\title{
Design Exploration of Energy-Performance Trade-Offs for Wireless Sensor Networks
}

\author{
Ivan Beretta ${ }^{1}$, Francisco Rincon ${ }^{2}$, Nadia Khaled ${ }^{3}$, \\ Paolo Roberto Grassi ${ }^{4}$, Vincenzo Rana ${ }^{4,1}$, David Atienza ${ }^{1}$ \\ ${ }^{1}$ ESL, École Polytechnique Fédérale de Lausanne, Switzerland, \{ivan.beretta, david.atienza\}@epfl.ch \\ ${ }^{2}$ DACYA, Universidad Complutense de Madrid, Spain, francisco.rincon@fdi.ucm.es \\ ${ }^{3}$ Bioanalytical Science Dept., Nestlé Research Center, Switzerland, nadia.khaled@rdls.nestle.com \\ ${ }^{4} \mathrm{DEI}$, Politecnico di Milano, Italy, \{grassi, rana\}@elet.polimi.it
}

\begin{abstract}
Wireless sensor networks (WNSs) are gradually evolving from a promising technology to a well-established reality in a large set of different domains. In order to fulfill the requirements of the specific scenario, a WSN must provide the right tradeoff between performance and lifetime, which is heavily determined by the network design. However, although the complexity of WSNs is increasing, the design space exploration is often carried out manually without the support of a general analytical methodology. In this paper, we advocate a model-based approach as an efficient and scalable way to explore the energy-performance tradeoffs during the design. In particular, we show that it is possible to define systemlevel models to describe wide classes of WSNs, providing a quick and accurate network evaluation. As a proof of concept, we propose a general model that describes the main characteristics of a class of WSNs for human health monitoring, and we apply it to a real case study. The results show that the energy-performance estimation error of the model never exceeds $1.74 \%$ compared to real data, while the evaluation time is reduced by up to 6 orders of magnitude with respect to an accurate network simulation.
\end{abstract}

\section{Categories and Subject Descriptors}

C.2.1 [Computer Communication Networks]: Network Architecture and Design; I.6.5 [Simulation and Modeling]: Model Development

\section{General Terms}

Design, Theory, Algorithms

\section{Keywords}

Wireless sensor networks, Cross-layer design, Model-based design, System-level modeling, Wireless body sensor networks

Permission to make digital or hard copies of all or part of this work for personal or classroom use is granted without fee provided that copies are not made or distributed for profit or commercial advantage and that copies bear this notice and the full citation on the first page. To copy otherwise, to republish, to post on servers or to redistribute to lists, requires prior specific permission and/or a fee.

DAC 2012, June 3-7, 2012, San Francisco, California, USA.

Copyright 2012 ACM 978-1-4503-1199-1/12/06 ...\$10.00.

\section{INTRODUCTION}

In the last years, wireless sensor networks (WNSs) are becoming a well-established reality in many different domains, including military applications, environment control, industrial supervision and health monitoring [1]. In order to deal with the specific requirements of a given application domain, a WSN has to meet certain performance requirements as well as to guarantee a sufficient lifetime, which are often conflicting goals. The right tradeoff between these two objectives, as well as the prevention of undesired behaviors such as unbalanced performance among the different nodes of the WSN, can be guaranteed by accurately evaluating the network configurations during the design phase. In order to help the designer during the energy-performance tradeoff analysis, many design space exploration (DSE) techniques for WSNs have been proposed in the literature [3][4], and most of the classic optimization algorithms can also be adapted to WSNs with a low effort. However, providing such algorithms with an accurate system-level estimation of the WSN performance is still an open problem, and it is necessary to correctly lead the DSE algorithm to the detection of the Pareto-optimal network configurations.

The evaluation of a particular WSN includes aspects that span across multiple layers (from the network to the hardware, to the application level), and it can be performed in three ways [6]: a set of physical experiments, a network simulation or an analytical model. However, when a large number of configurations needs to be evaluated during the DSE phase, both the empirical experiments and the simulation become impractical, as the former cannot be automated, while the latter takes an unacceptable amount of time. Conversely, the analytical model enables a fast evaluation and a deep understanding of the dynamics of the network, but its definition raises several challenges related to its accuracy and reusability. In fact, a detailed characterization of a specific WSN has been shown to lead to efficient network designs [5], but such a model requires a deep knowledge about the application and the target platform, and it cannot be reused to model different classes of WSNs. On the other hand, a generic system-level model that can be easily instantiated to a specific WSN would greatly simplify the task of the designer, but no model with these characteristics has been proposed yet, as it is complex to define a characterization that can describe all the different classes of WSNs with a sufficient accuracy.

Although the definition of a general model is limited by 
the great differences among the WSN domains, in this paper we aim at showing that it is possible to focus the scope of the model to wide classes of networks in order to capture their most relevant aspects, thus providing a model that is both detailed and reusable on many instances of WSNs. This work shows that a multi-layer characterization of the nodes and of their interactions in a well-defined class of WSNs leads to an accurate estimation with respect to both real and simulated data, and that a DSE algorithm greatly benefits from a model-based evaluation in terms of execution time. Furthermore, in order to provide a coherent systemlevel estimation of the network during the DSE, we propose a set of performance metrics that belong to different layers (i.e., delay, application quality, energy consumption), which lead to the determination of the optimal energy-performance tradeoffs. As a proof of concept, the model we propose targets the wide class of wearable wireless body sensor networks (WBSNs), which are a rising technology in the field of human health monitoring [2] both for medical and personal use. Experimental evaluations conducted on a real-world WBSN show that the proposed model never generates an estimation error greater than $1.74 \%$.

\section{RELATED WORK}

Over the last years, model-based evaluation as a support for DSE has been extensively explored in many fields. In the WSN domain, node and network models have been traditionally proposed to characterize specific aspects of the network, and to validate new protocols [14] or energy management strategies [3]. None of them, however, guarantees a general system-level description that can be easily adapted to describe a real WSN.

At the node level, analytical models for all the most common hardware blocks were proposed even before the advent of WSNs. In particular, detailed energy characterizations are available for hardware circuits, sensors and microcontrollers [8], memory banks [7], and radio circuits [9][15]. However, these models do not consider any interdependency between the different parts of the system, hence they are not sufficient to describe the behavior of a set of networked nodes. In [3], the authors relate the energy consumption and the throughput of the node to the supply voltage of the microcontroller and the modulation level of the radio. Although the work is a good example of how different aspects of the node (i.e., sensing, processing and transmission) can be combined, the parameters that are considered are only a small subset of the ones that can be found on real nodes.

At the network level, several works propose a model of different media access control (MAC) protocols, and in particular the widely-adopted IEEE 802.15.4 [16] standard. For example, [10] characterizes the behavior of the IEEE 802.15.4 MAC layer on large-scale networks, both in terms of energy consumption and packet transmission probability. In [11], a similar analysis is proposed for WBSNs, with a particular emphasis on the radio activity of the node. The works in [17] and [18] focus on the part of the IEEE 802.15.4 standard that works in TDMA mode, and propose two separate techniques to estimate the expected packet delay. However, none of the aforementioned network models propose an in-depth analysis of the application executed by the nodes, which is crucial to have a coherent global evaluation of the WSN.

Another important aspect of the trade-off analysis is the definition of a set of metrics that capture all the relevant dy-

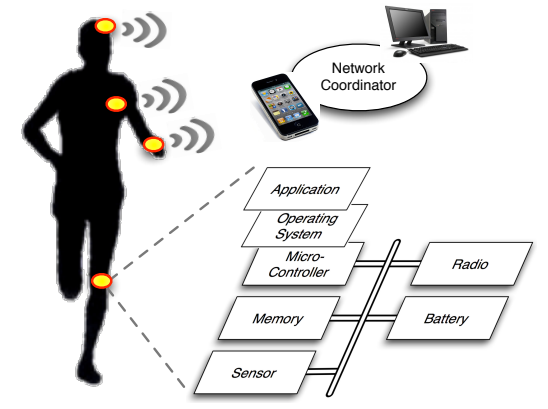

Figure 1: Overview of a typical WBSN

namics of the network. Traditionally, energy consumption is always a major concern during the network evaluation [14][3][10], but other metrics such as throughput and endto-end delay may be considered. For example, different energy/delay tradeoffs are explored as a function of the voltage and the radio modulation in [26]. However, no applicationrelated metric is generally proposed in order to characterize the overall behavior of the network as seen by the end user.

As a conclusion, none of the existing models provides a coherent system-level description that can be applied to realworld WSNs, mainly because they only focus on specific aspects of the system and often neglect the final application. In our work, we show that a general -and yet reliableanalytical model for the nodes and the whole network can be defined if its scope is limited to a set of WSNs sharing similar structures and application domains.

\section{SYSTEM-LEVEL MODEL FOR WBSNS}

In this section, we propose a general system-level model that characterizes the most important multi-layer interdependencies of the networks belonging to the same domain. As a proof of concept, we target the WBSN domain.

\subsection{WBSN Domain Analysis}

A typical WBSN [12] follows the structure illustrated in Figure 1. The network comprises a set of low-power nodes that can be worn by the same person or by different ones (e.g., the patients in a hospital, or a team of athletes) to monitor one or more vital signs to be sent to a central network coordinator. Once the signal has been sensed, each node performs a data pre-processing using a software application executed on a microcontroller-based hardware architecture (see Figure 1), and finally sends the output to the coordinator through the wireless channel. The coordinator is responsible for the analysis of the data, and the definition of the network activity (e.g., the enforcement of the MAC protocol). In WBSNs, a star topology network is generally employed, hence the communication between a node and the coordinator is direct [12]. Moreover, the wireless channel is shared among the nodes using a collision-free, time-division multiple access (TDMA) policy, which leads to a lower energy consumption with respect to a contention access. These assumptions are sufficient to characterize a wide set of networks in the WBSN domain, and they enable us to define an abstract model that can be easily adapted to real nodes and standards, as we show later by means of a case study.

\subsection{Network Model}

In the network model, we capture the interactions among 
the nodes and, in particular, how they share the wireless channel. For this purpose, the $N$ nodes of the network are now considered as black boxes generating an output stream of $\phi_{\text {out }}$ bytes per second $(\mathrm{B} / \mathrm{s})$. The transmission is regulated by the MAC protocol, which aims at assigning a transmission interval $\Delta_{t x}^{(n)}$ (the index denotes that the quantity refers to node $n$ ) per second to each node, by acting on protocol-specific parameters that form a configuration $\chi_{\text {mac }}$. Each node is then in charge of tuning the throughput $\phi_{\text {out }}^{(n)}$ in order to be able to deliver its data in the time $\Delta_{t x}^{(n)}$.

To describe the MAC layer, we introduce the following abstractions that capture its most recurring characteristics:

- a data overhead due to packetization and flow control, consisting of a number of extra bytes that are required to transmit $\phi_{\text {out }}$ (e.g., headers and tails). We indicate this overhead as $\Omega\left(\phi_{\text {out }}, \chi_{\text {mac }}\right)$ (measured in $\mathrm{B} / \mathrm{s}$ );

- a control overhead, which includes the control messages (e.g., synchronization packets and acknowledgements) that are exchanged between a node and the coordinator. As we further detail in Section 3.3, these messages generate an energy dissipation due to their transmission/reception. We identify the volume of control messages from the coordinator to the node and vice versa as $\Psi_{c \rightarrow n}\left(\chi_{m a c}\right)$ and $\Psi_{n \rightarrow c}\left(\chi_{m a c}\right)$ (measured in $\mathrm{B} / \mathrm{s}$ );

- a timing overhead per second, i.e., time intervals where the channel is unavailable, either because of the transmission of control messages or because the network is kept idle. We call this quantity $\Delta_{\text {control }}\left(\chi_{m a c}\right)$;

- a time discretization. Since a protocol does not generally assign an arbitrary and continuous transmission time to each node. We define $\delta$ as the base time unit that is used in the selected protocol, and we express the transmission intervals as multiples of $\delta$.

The goal of the network design is to size the transmission intervals to enable each node to deliver all its data and the corresponding control information. We model this as an assignment problem that is tailored for the typical startopology TDMA transmission of WBSNs, but it can be also adapted to a contention access protocol (in fact, the $\Delta_{t x}^{(n)}$ 's can be statistically determined as the average amount of time a node can successfully transmit per second, as shown in [19] for the CSMA/CA). In particular, the MAC protocol has to find a number $k^{(n)}$ for each node $n$ such that:

$$
\Delta_{t x}^{(n)}=k^{(n)} \cdot \delta \geq T_{t x}\left(\phi_{\text {out }}^{(n)}+\Omega\left(\phi_{\text {out }}^{(n)}, \chi_{\text {mac }}\right)\right),
$$

where $T_{t x}(\cdot)$ denotes the transmission time required to send the specified amount of data, and depends on the physical radio. Additionally, the assignment of the transmission intervals by the MAC protocol must be constrained in order not to exceed the total of one second:

$$
\sum_{i=1}^{N} \Delta_{t x}^{(i)}+\Delta_{\text {control }}\left(\chi_{\text {mac }}\right)=1 .
$$

From the DSE perspective, allowing the network to stay silent for a long time leads to good solutions in terms of energy consumption, but in practice it increases the data delay. Hence, we define the delay function $d\left(\chi_{\text {mac }}\right)$ to quantify the average (or the maximum) time between the generation of the data and the instant it is received by the coordinator. Such a function cannot be defined in the general case, but it can be determined according to the specific MAC and the traffic patterns of the nodes, as we show in the case study.

\subsection{Node Model}

A typical WBSN node follows the microcontroller-based architecture shown in Figure 1. We hereby propose a model that captures the interdependency among the hardware components in terms of consumption and application-related metrics, as well as the influence of the network configuration on the single node. We characterize the node by means of a configuration $\chi_{\text {node }}$, which includes the configurable parameters both on the hardware side (e.g., frequency, transmission power), and on the software side. All the parameters that cannot be tuned, or that are not relevant for a system-level optimization, will not be detailed in this model.

The node first samples the physiologic signal with a frequency $f_{s}$, and the samples are then quantized by an A/D converter to produce values of $L_{a d c}$ bytes, thus generating an input stream $\phi_{i n}$ of $f_{s} \cdot L_{a d c}(\mathrm{~B} / \mathrm{s})$. The sampling activity leads to an energy dissipation that can be expressed as:

$$
E_{\text {sensor }}=E_{\text {transducer }}+\left[\alpha_{s, 1} \cdot f_{s}+\alpha_{s, 0}\right] .
$$

A linear function of $f_{s}$ (with coefficients $\alpha_{s, 1}$ and $\alpha_{s, 0}$ ) captures the behavior of the A/D circuit [21], whereas $E_{\text {transducer }}$ is an overhead included by the transducer.

The input stream $\phi_{i n}$ is then processed by an application, which typically consists of filtering or data compression. The behavior of the application layer is determined by a set of parameters (e.g., approximation factors and compression ratios), which determine three key aspects:

- the output stream $\phi_{\text {out }}$. From a quantitative perspective, the application can be modeled as a function $h$ that processes the input stream $\phi_{i n}$ and produces a certain amount of results to be transmitted. As a consequence, the output of the node is equal to $\phi_{\text {out }}=$ $h\left(\phi_{\text {in }}, \chi_{\text {node }}\right)$ and, if an estimation of the transmission errors is available (e.g., [9]), then the average amount of retransmitted data can be added to the original $\phi_{\text {out }}$;

- the resource usage. To represent these quantities, we define a vector $\mathbf{u}=\left(\right.$ Duty $\left._{a p p}, M_{a p p}, \gamma_{a p p}, u_{4}, \ldots, u_{n}\right)$ that contains $n$ elements, one for each hardware resource that can be tuned on the target platform. We use a different notation to identify the duty cycle of the application on the microcontroller (Dutyapp), the amount of memory required during the execution $\left(M_{a p p}\right)$, and the number of memory accesses $\left(\gamma_{a p p}\right)$, which will be used later for energy considerations. The resource usage depends on how the node is tuned (i.e., $\chi_{\text {node }}$ ) and on the amount of data to be processed. Hence, we can define a function vector $\mathbf{k}=\left(k_{1}, \ldots, k_{n}\right)$ such that $\mathbf{u}=\mathbf{k}\left(\phi_{\text {in }}, \chi_{\text {node }}\right)$, where $k_{i}\left(\phi_{\text {in }}, \chi_{\text {node }}\right)$ computes the usage of resource $i$.

- the output quality. As the application generally introduces an approximation, we define an applicationspecific function $e\left(\phi_{i n}, \chi_{\text {node }}\right)$ that measures the loss of quality between the original and the transmitted data.

After characterizing the application, we focus on the effects of its execution. On the microcontroller side, the execution generates an energy dissipation that linearly depends on the duty cycle and on the operating frequency $\left(f_{\mu C}\right)[21]$ :

$$
E_{\mu C}=\text { Duty }_{\text {app }} \cdot\left[\alpha_{\mu C, 1} \cdot f_{\mu C}+\alpha_{\mu C, 0}\right] .
$$


The execution also leads to an energy consumption due to memory access, which can be estimated as follows [7]:

$$
E_{m e m}=\gamma_{a p p} T_{m e m} \cdot E_{a c c}+\left(1-\gamma_{a p p} T_{m e m}\right) 8 M_{a p p} \cdot E_{i d l e}^{b i t} .
$$

This equation includes two contributions: a dynamic consumption due to the $\gamma_{a p p}$ memory accesses, and a residual that occurs during idle periods and is proportional to the memory size. In the equation, $T_{m e m}$ indicates the access time, $E_{a c c}$ defines the consumption of a single access, and $E_{\text {idle }}^{\text {bit }}$ denotes the dissipation per bit due to leakage.

Finally, the output stream $\phi_{\text {out }}$ and the control information need to be transmitted to the coordinator by the radio unit during the assigned transmission intervals. The physical radio determines the transmission time in Equation (1) and the dissipation associated to the reception $\left(E_{r x}\right)$ and the transmission $\left(E_{t x}\right)$ of one bit, the latter being related to the power of the carrier signal [9], which must be chosen to achieve a low packet error rate. Thus, the energy consumption due to the radio can be expressed as:

$$
\begin{aligned}
E_{\text {radio }}= & {\left[8\left(\phi_{\text {out }}+\Omega\left(\phi_{\text {out }}, \chi_{\text {mac }}\right)\right)+8 \Psi_{n \rightarrow c}\left(\chi_{\text {mac }}\right)\right] \cdot E_{\text {tx }} } \\
& +8 \Psi_{c \rightarrow n}\left(\chi_{\text {mac }}\right) \cdot E_{r x} .
\end{aligned}
$$

Then, after including the contribution of all the analyzed layers, the overall node consumption can be expressed as:

$$
E_{\text {node }}=E_{\text {sensor }}+E_{\mu C}+E_{\text {mem }}+E_{\text {radio }} .
$$

\subsection{System-Level Evaluation Metrics}

To complete the description of the WBSN, we combine the performance metrics of each node (i.e., $E_{\text {node }}$ and $\left.e\left(\phi_{\text {in }}, \chi_{\text {node }}\right)\right)$ into consistent network-level objective functions.

As mentioned in Section 1, finding balanced configurations is a major concern while combining the different metrics, in order to avoid situations where the coordinator receives data of different quality, or where heavily optimized nodes are alternated to other nodes with an insufficient lifetime. As a consequence, we define the network-level energy consumption $\left(E_{n e t}\right)$ as a weighted combination of the average energy consumption of the nodes, and the sample standard deviation of this quantity over the WBSN:

$E_{\text {net }}=\sum_{i=1}^{N} \frac{E_{\text {node }}^{(n)}}{N}+\vartheta \cdot \sqrt{\frac{1}{N-1} \sum_{i+1}^{N}\left[E_{\text {node }}^{(n)}-\sum_{i=1}^{N} \frac{E_{\text {node }}^{(n)}}{N}\right]^{2}}$,

where $\vartheta$ is a positive constant that determines the importance of the balance among the nodes.

We can define a network-level application quality metric in a similar way, by combining all the loss-of-quality functions $e^{(n)}\left(\phi_{i n}, \chi_{\text {node }}\right)$ as we did in Equation (8) for $E_{\text {node }}^{(n)}$.

\section{A REAL-WORLD WBSN CASE STUDY}

In this section, we show that the proposed multi-layer model for WBSNs can be easily used to model a real network that uses a commercial platform and widespread standards.

\subsection{Case Study Overview}

We propose an illustrative case study of a WBSN for electrocardiography (ECG) monitoring. We envision a scenario that can take place in a hospital, where $N$ patients (in this example, $N=6$ ) are wearing a node that is connected to a central base station. The nodes reduce the size of the output

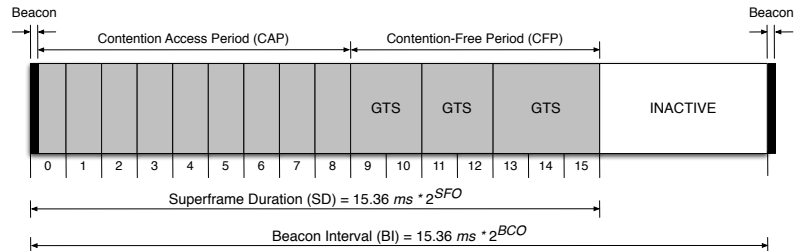

Figure 2: Structure of the IEEE 802.15.4 superframe

stream by applying one of the two available data compression techniques, i.e., digital wavelet transform (DWT) [23] and compressed sensing (CS) [13]. The two techniques have different properties in terms of complexity, signal quality and hardware requirements: for the sake of illustration, we assume that half of the nodes employ DWT, and the remaining ones execute CS.

As a node, we employ the Shimmer commercial platform [24], which includes an ultra low-power microcontroller, $10 \mathrm{kB}$ of RAM memory, and an IEEE 802.15.4 [16] radio module. The transmission is performed using the beaconenabled mode of the IEEE 802.15.4 MAC layer [16]. Considering the set of parameters on the node and the MAC protocol, the number of possible network configurations of this case study exceeds the tens of millions, thus making a deep DSE impractical by using network simulation or by collecting experimental data. The proposed model, on the other hand, contains all the structures that are needed to fully describe the target network.

\subsection{IEEE 802.15.4 Network Model}

We hereby show how the proposed system-level WBSN model can capture the relevant dynamics of the beaconenabled mode of the IEEE 802.15.4 [16] MAC protocol. In this MAC, a beacon is periodically sent by the coordinator to define the structure of the next superframe, a time interval whose structure is shown in Figure 2. The superframe is divided into an inactive and an active part, the latter being divided into 16 slots, 7 of which (known as guaranteed time slots, GTSs) are granted using a TDMA-like protocol.

The IEEE 802.15.4 MAC configuration is defined as $\chi_{\text {mac }}=$ $\left\{L_{\text {payload }}, S F O, B C O, \Delta_{t x}^{(1)}, \ldots, \Delta_{t x}^{(N)}\right\}$, where $L_{\text {payload }}$ is the payload in a data packet, and $S F O$ and $B C O$ denote the superframe and the beacon orders, which in turn determine the interval between two beacons $(B I)$ and the duration of the active part $(S D)$ (see Figure 2) [16]. Finally, the $\Delta_{t x}^{(n)}$ 's indicate the transmission time allocated to each node.

The IEEE 802.15.4 MAC protocol can be easily mapped on the structures we identified in Section 3.2. For example, the data overhead introduced by the MAC is equal to 13 bytes ( 11 for the header, 2 for the checksum) for each packet, hence $\Omega\left(\phi_{\text {out }}^{(n)}, \chi_{\text {mac }}\right)=13 \cdot \phi_{\text {out }}^{(n)} / L_{\text {payload }}$. In terms of control overhead, the protocol does not require any control message from node (thus $\Psi_{n \rightarrow c}\left(\chi_{\text {mac }}\right)=0$ ), whereas the coordinator sends a number of beacons (of variable length, which we denote as $L_{\text {beacon }}$ ) that depends on the number of superframes per second (i.e., $1 / B I$ ), and an acknowledgment ( 4 bytes) for each transmitted packet, thus $\Psi_{c \rightarrow n}\left(\chi_{\text {mac }}\right)=4 \cdot \phi_{\text {out }}^{(n)} / L_{\text {payload }}+L_{\text {beacon }} / B I$. Furthermore, $\Delta_{\text {control }}\left(\chi_{\text {mac }}\right)$ is the time required by the coordinator to transmit $1 / B I$ beacons per second, plus at least 9 slots reserved for contention access (which are not exploited in this case study), and the inactive period of the superframes. 
As we mentioned in Section 3.2, the model can handle additional protocol-specific constrains on the assignment of the $\Delta_{t x}^{(n)}$ 's. Firstly, the $\Delta_{t x}^{(n)}$ 's cannot be arbitrarily assigned because of the time discretization imposed by the slots. Hence, we define the base transmission time $\delta$ as the slot length, i.e., $S D / 16$, and we express all the $\Delta_{t x}^{(n)}$ 's as multiples of $\delta$. Then, as the protocol specifies that at most 7 slots can be used as GTSs, we formulate a constraint on the overall transmission time that can be allocated for the nodes, i.e., $\sum_{i=1}^{N} \Delta_{t x}^{(i)} \leq 7 / 16 \cdot S D / B I$.

Finally, thanks to the nature of data compression that leads to a uniform output rate, a simple delay model (based on the one in [17]) can be formulated. In particular, the worst-case delay for a node $n$ occurs when the remaining nodes use all their slots (and the control overhead for all the corresponding frames) before node $n$ is enabled to transmit:

$$
d^{(n)}\left(\chi_{m a c}\right) \leq \sum_{i=1, i \neq n}^{N} \Delta_{t x}^{(i)}+\left\lceil\frac{1}{7} \sum_{i=1, i \neq n}^{N} \Delta_{t x}^{(i)}\right\rceil \Delta_{\text {control }}
$$

\subsection{Shimmer Node Model}

In this section, we apply the node model described in Section 3.3 to the Shimmer platform [24]. As the node platform is already implemented, some parameters are fixed. In particular, the sampling frequency is determined by the nature of the ECG signal and is fixed to $f_{s}=250 \mathrm{~Hz}$, and the resolution $L_{A D C}$ of the A/D converter is set to $12 \mathrm{bits}$, thus generating a constant input stream $\phi_{i n}=375 \mathrm{~B} / \mathrm{s}$. The contribution of the $10 \mathrm{kB}$ memory block is also constant, as the memory accesses are determined by the Shimmer-specific implementations of the DWT and CS algorithms [13]. At the radio level, the power of the carrier signal has been set to a sufficient level in order to minimize the probability of a packet error, thus avoiding an increment of $\phi_{\text {out }}$ due to retransmission. Hence, the configuration of a node is characterized as $\chi_{\text {node }}=\left\{C R, f_{\mu C}\right\}$, where $C R$ is the compression ratio, and $f_{\mu C}$ is the frequency of the microcontroller.

The output stream $\phi_{\text {out }}$ can be easily expressed as a function of $C R$, i.e., $\phi_{\text {out }}=h\left(\phi_{\text {in }}, \chi_{\text {node }}\right)=\phi_{\text {in }} \cdot C R$, which holds for both the DWT and the CS applications. However, the two compressions show different duty cycles and loss-of-quality functions. The duty cycle of the Shimmer implementations of DWT and CS show a marginally dependency on $C R$, but there is a relation with respect to $f_{\mu C} \in \chi_{\text {node }}$. By analyzing the execution, we can define the resource usage function as $\mathbf{k}\left(\phi_{i n}, \chi_{\text {node }}\right)=\left(k_{D W T}, k_{C S}\right)=$ $\left(2265.6 / f_{\mu C}, 388.8 / f_{\mu C}\right)$. To estimate the quality of the application, we select the percentage root-mean-square difference $(P R D)$ [13], which quantifies the difference between the original ECG and the one reconstructed by the coordinator. Although the actual $P R D$ value can only be determined by measuring or simulating the actual reconstructed signal, we computed an analytical estimation using two fifth-order polynomial functions $P_{5}^{(D W T)}(C R)$ and $P_{5}^{(C S)}(C R)$ that fit the experimental data provided in [13].

\section{EXPERIMENTAL RESULTS}

In this section, we evaluate the accuracy of the proposed model-based estimation with respect to experimental data. The results refer to the case study discussed in Section 4, but tests on different networks show a similar accuracy.

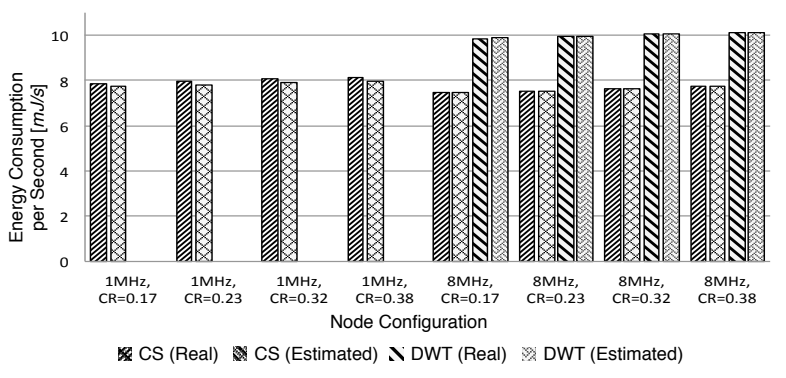

Figure 3: Estimation of the node consumption with different configurations

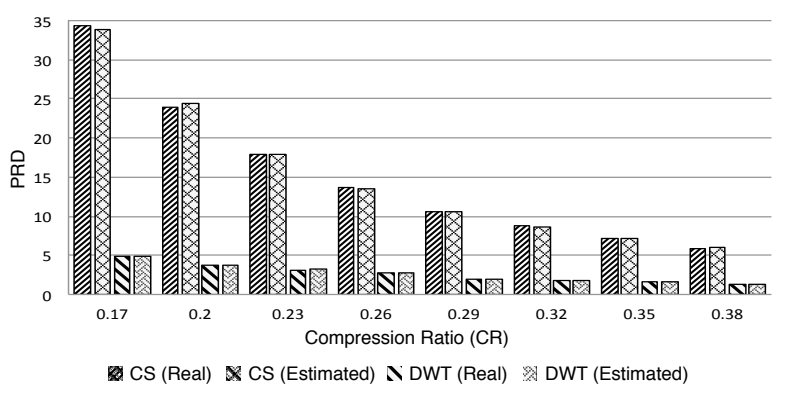

Figure 4: Estimation of the application behavior by means of the $P R D$ metric

\subsection{Estimation Accuracy}

The first set of experiments aims at validating the estimation provided by the model. We first validated the model equations with respect to real experimental data obtained under different operating conditions. Figure 3 shows the estimation of the overall energy consumption of the nodes with set of realistic configurations $\chi_{\text {node }}$. The energy estimation proves to be very accurate, as the average error on all the $f_{\mu C}$ 's and $C R$ 's is equal to $0.88 \%$ for the CS, and to $0.13 \%$ for DWT, and the maximum error does not exceed $1.74 \%$. The model also predicts that the DWT cannot complete its execution with $f_{\mu C}=1 \mathrm{MHz}$ because its duty cycle exceeds $100 \%$. Figure 4 shows the estimation error for the PRD's, which proves to be very low $(0.92 \%$ for the CS, $0.46 \%$ for the DWT), thus showing that the model accurately estimates a crucial metric that can be exactly determined only by analyzing or simulating the actual compressed ECG.

In order to validate the network model, we compared the estimated delay to the results of a network simulation performed using the popular Castalia framework [25]. The choice of a network simulator over experimental data is justified by the possibility of deeply monitoring the packet flow. In spite of being a worst-case estimation, the delay function in Equation (9) provides an average overestimation lower than $100 \mathrm{~ms}$ over a set of 130 simulations with realistic $\phi_{\text {out }}$ 's and $\chi_{m a c}$ 's, which is acceptable in this application.

\subsection{Design Space Exploration Performance}

In this paragraph, we aim at validating the proposed modelbased evaluation within the context of the DSE. We employed the proposed WBSN model in a set of multi-objective optimization techniques, including genetic algorithms (which have been already used in the WSN domain [3]) and simulated annealing [27], without experiencing any relevant difference in terms of quality of the solutions. In terms of execution time, the proposed evaluation clearly outperforms 

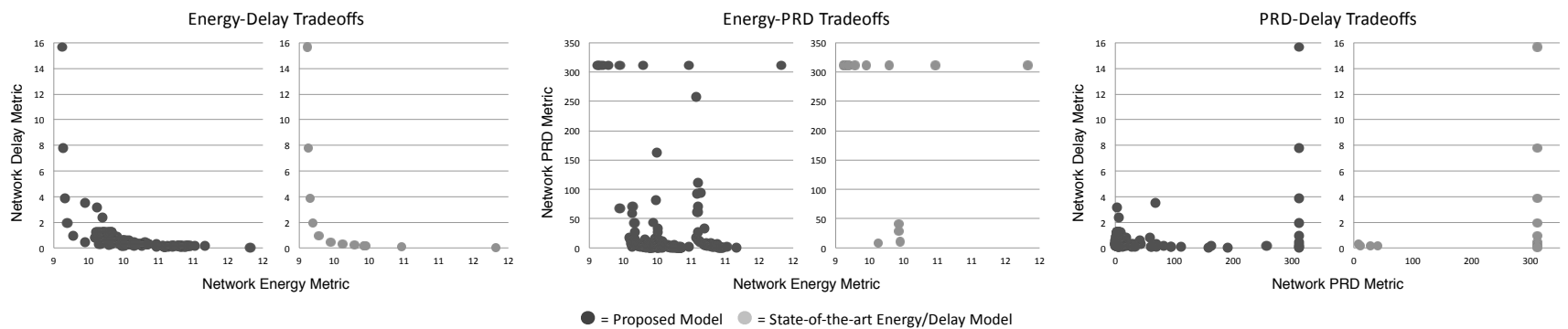

Figure 5: Tradeoffs detected using the proposed model and a state-of-the-art energy/delay model [26]

a complete network simulation, in fact, a network simulation takes 5 to 10 minutes in our case study, while the model can be evaluated approximately 4800 times per second.

Figure 5 shows the optimal tradeoffs between the three metrics we included in our model and, in order to underline the importance of considering all these metrics, the solutions are compared to the ones found by using a state-of-the-art energy/delay model [26]. It can be observed that the Pareto set generated according to the energy/delay model only contains a subset (i.e., approximately $7 \%$ ) of the tradeoffs that are found using the proposed model: this is due to the fact that the energy/delay model does not include an additional application-aware metric. As a consequence, it only approximates the energy/delay curve, but it does not allow the DSE algorithm to recognize the solutions that are optimal in terms of $P R D$. In order to detect the large number of Pareto tradeoffs characterized by acceptable mid-range $P R D$ 's, the proposed multi-layer model must be employed.

\section{CONCLUSION}

In this work, we have shown the benefits of a quick and accurate analytical evaluation in the context of model-based design of WSNs. Although WSNs show a wide range of different characteristics in different fields, it is possible to formulate abstract system-level models for broad classes of networks, which share common architectural and network structures, or more generally belong to the same domain. As a proof of concept, we have considered the class of WBSNs, and we have proved that a general and comprehensive model can be defined, and it can be applied to real networks with a low effort and a high accuracy. The results on a real case study show that the estimation error for energy and performance never exceeds $1.74 \%$ with respect to real data, while the estimation time is up to six orders of magnitude lower than an evaluation performed by a network simulator.

\section{ACKNOWLEDGMENTS}

This research was partially supported by the Swiss National Science Foundation (SNF), under grant 200021-127282, and by the Spanish Government under grant TIN2008-00508.

\section{REFERENCES}

[1] J. Yick et al., "Wireless sensor network survey," Computer Networks, no. 52, 2008, pp. 2292-2330.

[2] M. Patel and J. Wang, "Applications, challenges, and prospective in emerging body area networking technologies," Wireless Communications, vol. 17, no. 1, pp. 80-88, 2010.

[3] C.-t. Yeh et al., "Energy-Aware Data Acquisition in Wireless Sensor Networks," in Proc. of IMTC, 2007, pp. 1-6.

[4] S. Nabar et al. , "Minimizing Energy Consumption in Body Sensor Networks via Convex Optimization," in Proc. of $B S N$, 2010, pp. 62-67.
[5] Z. He et al., "Energy-aware portable video communication system design for wildlife activity monitoring," IEEE Circuits and Systems Magazine, vol. 8, no. 2, pp. 25-37, 2008.

[6] L. S. Bai et al., "Automated construction of fast and accurate system-level models for wireless sensor networks," in Proc. of DATE, 2011, pp. 1-6.

[7] H. Koc et al., "Minimizing Energy Consumption of Banked Memories Using Data Recomputation," in Proc. of ISLPED, 2006, pp. 358-361.

[8] V. Gutnik and A. P. Chandrakasan, "Embedded power supply for low-power DSP," IEEE Trans. on Very Large Scale Integration (VLSI) Systems, vol. 5, no. 4, pp. 425-435, 1997.

[9] C. Schurgers et al., "Power management for energy-aware communication systems," ACM Trans. on Embedded Computing Systems, vol. 2, no. 3, pp. 431-447, 2003.

[10] M. Kohvakka et al., "Performance analysis of IEEE 802.15.4 and ZigBee for large-scale wireless sensor network applications," in Proc. of PE-WASUN, 2006, pp. 48-57.

[11] N. F. Timmons and W. G. Scanlon, "Analysis of the performance of IEEE 802.15.4 for medical sensor body area networking," in Proc. of SECON, 2004, pp. 16-24.

[12] S. Ullah et al., "A Comprehensive Survey of Wireless Body Area Networks," Journal of Medical Systems, pp. 1-30, 2010.

[13] H. Mamaghanian et al., "Compressed Sensing for Real-Time Energy-Efficient ECG Compression on Wireless Body Sensor Nodes," Trans. on Biomedical Engineering, no. 99, p. 1, 2011.

[14] P. Suarez et al., "Increasing ZigBee network lifetime with X-MAC," in Proc. of REALWSN, 2008, pp. 26-30.

[15] Casilari et al., "Modeling of Current Consumption in 802.15.4/ZigBee Sensor Motes," Sensors, vol. 10, no. 6, pp 5443-5468, 2010

[16] IEEE, IEEE Std 802.15.4-2006, 2006.

[17] A. Koubaa et al., "GTS allocation analysis in IEEE 802.15.4 for real-time wireless sensor networks," in Proc. of IPDPS, 2006, pp. 8.

[18] P. Park et al., "Performance Analysis of GTS Allocation in Beacon Enabled IEEE 802.15.4," in Proc. of SECON, 2009, pp. $1-9$.

[19] C. Buratti, "Performance Analysis of IEEE 802.15.4 Beacon-Enabled Mode," IEEE Trans. on Vehicular Technology, vol. 59, no. 4, pp. 2031-2045, 2010.

[20] R. Rieger and J. T. Taylor, "An Adaptive Sampling System for Sensor Nodes in Body Area Networks," IEEE Trans. on Neural Systems and Rehabilitation Engineering, vol. 17, no. 2, pp. 183-189, 2009.

[21] T. Burd and R. Brodersen, "Energy efficient CMOS microprocessor design," in Proc. of HICSS, 1995, pp. 288-297.

[22] F. Rincón et al., "Development and Evaluation of Multilead Wavelet-Based ECG Delineation Algorithms for Embedded Wireless Sensor Nodes," IEEE Trans. on Information Technology in Biomedicine, vol. 15, no. 6, pp. 854-863, 2011.

[23] R. Benzid et al., "Fixed percentage of wavelet coefficients to be zeroed for ECG compression," Electronics Letters, vol. 39, no. 11 , pp. 830-831, 2003.

[24] A. Burns et al., "SHIMMER - A Wireless Sensor Platform for Noninvasive Biomedical Research," IEEE Sensors Journal, vol. 10 , no. 9 , pp. 1527-1534, 2010.

[25] Castalia Simulator, http://castalia.npc.nicta.com.au/.

[26] G. Kumar et al., "End-to-End Energy Management in Networked Real-Time Embedded Systems," Trans. on Parallel and Distributed Systems, vol. 19, no. 11, pp. 1498-1510, 2008.

[27] D. Nam and C. H. Park, "Multiobjective Simulated Annealing: A Comparative Study to Evolutionary Algorithms," Intl. Journal of Fuzzy Systems, vol. 2, no. 2, pp. 87-97, 2000. 